\title{
Oposición en Twitter como novedad en las elecciones seccionales 2019 en Ecuador
}

Opposition on Twitter as a novelty in the 2019 sectional elections in

\section{Ecuador}

Herrería-Benavides, Ana-Cristina

Pontificia Universidad Católica del Ecuador Sede Ibarra (PUCESI)

crisherrería@gmail.com

Pozo-Vilca, Sandra

Pontificia Universidad Católica del Ecuador Sede Ibarra (PUCESI)

pozo.mari@gmail.com

Márquez-Domínguez, Carmelo

carmelomarquez@gmail.com

Forma de citar este artículo:

Herrería-Benavides, A. C., Pozo-Vilca, S., y Márquez-Domínguez, C. (2020). “Oposición en Twitter como novedad en las elecciones seccionales 2019 en Ecuador", RAEIC, Revista de la Asociación Española de Investigación de la Comunicación, vol. 7, núm. 13, 250-271

https://doi.org/10.24137/raeic. 7.13.12

RAEIC, Revista de la Asociación Española de Investigación de la Comunicación vol. 7 , núm. 13 (2020), 250-271 


\section{Resumen:}

En las elecciones seccionales del 24 de marzo del 2019, en Ecuador, se eligieron 11.069 dignidades de elección popular entre principales y suplentes. En dos años de Gobierno de Lenin Moreno, no se ha identificado una oposición desde los territorios o los medios de comunicación tradicionales y la comunicación digital han marcado una agenda mediática pasiva. Las elecciones del 2019 presentaron un panorama diferente para el ejecutivo y esto se debe al involucramiento del expresidente Rafael Correa, quien actualmente es la cara de la oposición en redes a través de la red social Twitter acompañando al partido político Fuerza Compromiso Social. Para la redacción de esta investigación, se aplicó una metodología mixta, en lo que refiere al método analítico para, con herramientas de observación, recopilar los tuits generados durante los 45 días la campaña y, mediante el método sintético, se seleccionaron los resultados de las elecciones seccionales de la página oficial del Consejo Nacional Electoral con el fin de conocer cómo ha influenciado una red social en la campaña electoral ecuatoriana y, desde esta perspectiva, reconocer la realidad de la democracia tras las elecciones seccionales de marzo 2019 en Ecuador. Sobre los candidatos, se priorizaron las candidaturas de Prefecturas y los candidatos que se presentaron a elecciones para el Consejo de Participación Ciudadana y Control Social. Los medios de visualización de las candidaturas no son equitativos y se construyen a través de las redes sociales. En el caso del partido político FCS, se fortalece en los espacios digitales puesto que, tras la campaña, consiguió mantener su presencia como partido en el registro electoral del CNE dado que obtuvo el $5 \%$ del total del electorado empadronado (porcentaje mínimo requerido para presentarse a los comicios del 2021).

Palabras clave: oposición, comunicación, política, campaña, tecnología.

\section{Abstract:}

In the regional elections of March 24, 2019, in Ecuador, 11,069 popularly elected dignitaries were chosen among main and alternate candidates. In the two years of Lenin 
Moreno's government, no opposition has been identified from the territories or the traditional media, and digital communication has marked a passive media agenda. The 2019 elections presented a different panorama for the executive and this is due to the involvement of former president Rafael Correa, who is currently the face of the opposition in social networks utilizing Twitter and promoting the political movement Fuerza Compromiso Social (FCS). In this research, a mixed methodology was applied. The analytical method was used for compiling, with observational tools, the Tweets generated during the 45 days of the campaign. Also, using the synthetic method, the results of the regional elections from the official page of the Consejo Nacional Electoral (CNE), the National Electoral Council, were analyzed in order to know how a social network influenced the Ecuadorian electoral campaign and, from this perspective, to evaluate the state of democracy after the regional elections of March 2019 in Ecuador. About the candidates, FCS, also known as List 5, gave priority to the candidates of the Prefectures and the candidates who stood for election to the Consejo de Participación Ciudadana y Control Social (Council for Citizen Participation and Social Control). One of the main conclusions is that the means of visualization of the candidacies are not equitable and are constructed through social networks. In the case of FCS, this is strengthened in the digital realm since it maintained its presence as a party in the electoral registry of the CNE by obtaining $5 \%$ of the total registered electorate which is the minimum percentage required to participate in the 2021 elections.

Keywords: opposition, communication, politics, campaign, technology.

\section{INTRODUCCIÓN}

En las elecciones seccionales del 24 de marzo del 2019, en Ecuador se eligieron 23 prefectos y vice-prefectos, 221 alcaldes, 867 concejales urbanos, 438 concejales rurales, 4089 vocales de juntas parroquiales, en total 11.069 dignidades de elección popular entre principales y suplentes. Para el Gobierno Central el apoyo en los territorios es importante para seguir sus planes y proyectos programados a nivel nacional, según García Jurado (2000): “[...] la legitimidad política confiere a los gobiernos la facultad de 
exigir la obediencia política" (p.132), es decir, los ciudadanos se verán más cercanos a una autoridad local que a una nacional y en esta línea, la oposición que se ha identificado en la presente investigación, apuntó a ganar votos en lo local.

[...] las oportunidades que tiene la oposición de combatir la política de un gobierno dado son inciertas, pero no accidentales. Pero si las perspectivas de la oposición están determinadas principalmente por factores que escapan a su control inmediato, sí puede modificarse el grado de libertad que la oposición disfrute, mediante una elección deliberada (Dahl, 1997, p.193).

Durante los dos años del Gobierno de Lenin Moreno no se ha identificado una oposición desde los territorios. Las autoridades locales no han mostrado su apoyo y tampoco su desacuerdo al desarrollo de las políticas públicas implementadas desde el Gobierno Central. Los medios de comunicación tradicional y comunicación digital han marcado una agenda mediática pasiva, caracterizada por la mera transmisión de la información gubernamental, lo que nos ha significado inferir sobre la interpretación de Pasquino en su postura sobre el gobierno del pueblo, cuando se refiere que "la oposición es democracia". Y, de aquí, surge el cuestionamiento de análisis: ¿vivimos una realidad de dictadura perfecta? Esta pasividad nos motiva a reflexionar que "en las democracias modernas entonces pululan grupos contrapuestos y que compiten entre ellos, y que son relativamente autónomos en relación con el gobierno central. Los grupos representan intereses particulares aunque (por estrategia discursiva) los hagan ver como intereses nacionales" (Cante Cuervo y Franco Beatriz, 2014, p.11).

Gráfico 1. Surgimiento de la oposición en campaña Twitter.

Rafael Correa
"Quisieron enterrarnos, sin entender que somos semilla".
Vamos a participar en las elecciones de marzo.
La Revolución Ciudadana será listas 5.
Esta vez no es solo por personas, es por una causa.
¿Quieres recuperar la Patria y castigar a los traidores?
¡Todo, todito 5! pic.twitter.com/PDHljmE4HZ
O 10,3 mil 0.24 - 21 dic. 2018
Q 9.125 personas están hablando de esto


Fuente: Recopilación Diario El Comercio (2019).

Las elecciones del 2019 presentaron un panorama diferente para el ejecutivo, y esto se debe al involucramiento del expresidente Rafael Correa, quien actualmente es la cara de la oposición principalmente a través de la red social Twitter acompañando al movimiento político "Fuerza Compromiso Social" (FCS - Lista 5), fundado por un exministro de su gobierno. Afincarse en FCS le sirvió de colchón para la inscripción de los candidatos correístas y, desde una postura crítica y hasta de confrontación, expuso en esta red social y otros medios digitales su oposición al régimen de gobierno en Ecuador. El presente informe analizará la generación de la oposición desde la red social Twitter.

\section{METODOLOGÍA}

Para la redacción de esta investigación se aplicó una metodología mixta (cualicuantitativa). Los métodos analítico y sintético permitirán tomar, como referencia, postulados teóricos que referencian conceptos de oposición, espectacularización, agenda mediática, construcción de discurso, liderazgo, identificación partidista y videocracia para construir la historia del arte con investigaciones previas que refieren el tema del artículo. De esta investigación bibliográfica se podrá conocer la ruptura ideológica que tuvo Moreno por la cual fue calificado como "traidor" por Correa y posicionarse como uno de los mayores opositores del gobierno actual.

El análisis se plantea desde la importancia de la re-significación en la construcción del mensaje propagandístico tradicional expuesto en escenarios modernos como son las redes sociales y medios digitales, donde la vinculación permanente de los partidos y los medios como espacios de exposición pública son determinantes, y la ciudadanía como receptora de las propuestas. La espectacularización se mantiene en los mass media y la influencia de los spots de campaña en la construcción de la opinión pública, determina las características de impacto que estos deben tener respecto a "la estructura, el diseño, el contenido, la dirección y la estrategia, los temas, los participantes y los grupos sociales y finalmente las metáforas que se utilizaron" (García, 2017, p.161) 
En lo que refiere al método analítico, con la herramienta de análisis web FanPageKarma, se recopilaron los posteos generados durante los 45 días la campaña electoral, desde el 05 de febrero al 20 de marzo de 2019, en la cuenta @MashiRafael, donde se extrajeron los siguientes datos: número de post y repost, me gusta y comentarios. Se realizó también una desagregación de datos para conocer cuáles fueron las palabras más usadas y cuál fue su mensaje reiterativo para llamar a los votantes a elegir a sus candidatos.

Mediante el método sintético se recopilaron los resultados de las elecciones seccionales de marzo 2019 en Ecuador con los datos oficiales del Consejo Nacional Electoral (CNE), con el fin de conocer cómo queda el panorama electoral con lo que refiere al movimiento político "Fuerza Compromiso social" (lista 5) promocionado por el expresidente Rafael Correa. Con esos datos se arman las conclusiones de cómo ha influenciado la red social Twitter en la campaña electoral y desde esa perspectiva se puede establecer la viabilidad del tema del artículo que pretende brindar una respuesta a la realidad electoral en las elecciones seccionales de marzo 2019 en Ecuador.

\section{RESULTADOS}

El Para entender la importancia de los acontecimientos suscitados en las elecciones seccionales de marzo 2019 en Ecuador, se refiere el análisis sobre acción política de Paredes Goicochea, (2015) mediante los criterios de Hannah Arendt sobre acción política y acontecimiento, que se ajustan a la presente investigación:

Arendt muestra que existe una tensión entre el acontecer y el acontecimiento. El primero busca reducir la novedad a la tranquilidad de lo ya conocido, a lo acostumbrado, a lo que puede ser explicado e incluso calculado por sus antecedentes. El segundo, parte de lo ordinario para dar lugar a lo extraordinario, para romper el orden de la normalidad (p.29).

Sobre el acontecer nacional existe la idea del proceso electoral y los candidatos que se iban a postular por cada lista, el panorama respecto a la postura del actual gobierno frente a unas elecciones seccionales en el Ecuador, así como, las acciones típicas y 
ordinarias que de este proceso deberían resultar, sin embargo, alcanzar a vislumbrar el impacto de la oposición es vital para el estudio de la política.

Los resultados de la presente investigación del seguimiento de la red social Twitter: @MashiRafael, indican que durante el tiempo de campaña del 5 de febrero al 20 de marzo de 2019 el incremento en porcentaje desde el inicio es del 0,2\% en seguidores (5454). En la actualidad esta red social es una plataforma para los políticos y para hacer política por la interacción y la propagación que se genera con los seguidores de la cuenta. Sobre investigaciones previas se referencia a Gordillo Tejada (2019): "El exmandatario ecuatoriano sabe cómo transmitir su discurso en Twitter y cómo construir hilos de mensajes [...]" (p.84). Correa durante las elecciones seccionales 2019 en Ecuador hizo de su red social en twitter el medio para posicionar a: un nuevo partido político, apoyar a candidatos y mantener su oposición al Gobierno de Moreno.

Gráfico 2. Crecimiento en porcentaje de seguidores.

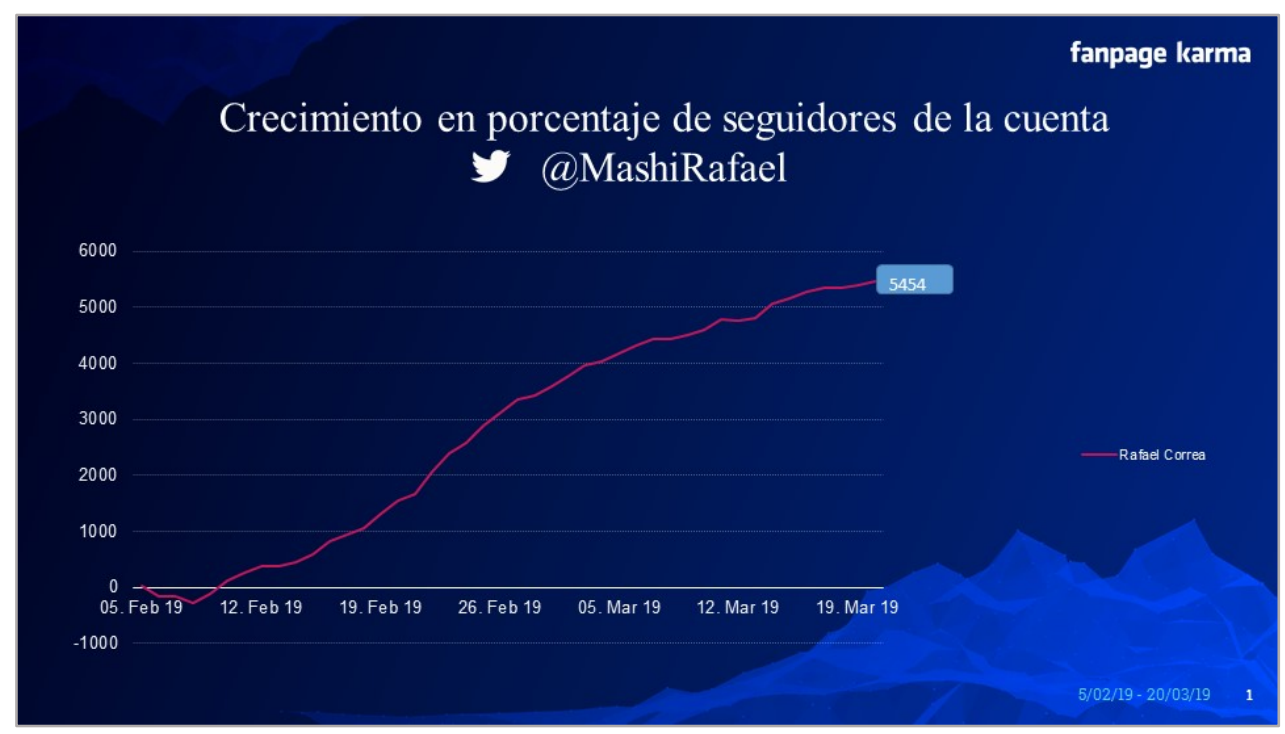

Fuente: Fanpagekarma y rediseñado por los autores

El exmandatario Correa no es nuevo en la actividad generada en redes sociales, durante sus diez años de gobierno, trabajó en la comunicación digital, para Villavicencio Salvador (2017) “[...] siendo pionero de la manipulación de la información política en el Ecuador a través de Twitter, fue capaz de conectarse con la gente [...]" (p.113). Durante la campaña electoral de 2019, Correa tuvo un promedio de 56 tuits diarios generando en 45 días 2.447 tuits promedio; en los cuales combinaba sus textos con imágenes, videos 
y links, de esta forma llegó a tener 573.646 retuits promedio de sus seguidores; es decir buscó llegar y mantenerse en el imaginario de los ciudadanos con el fin de posicionar su mensaje en este caso electoral (llamaba a votar por los candidatos de su partidos) y de oposición al actual gobierno.

Gráfico 3. Tuits y retuits durante los 45 días de campaña electoral de la cuenta @MashiRafael.

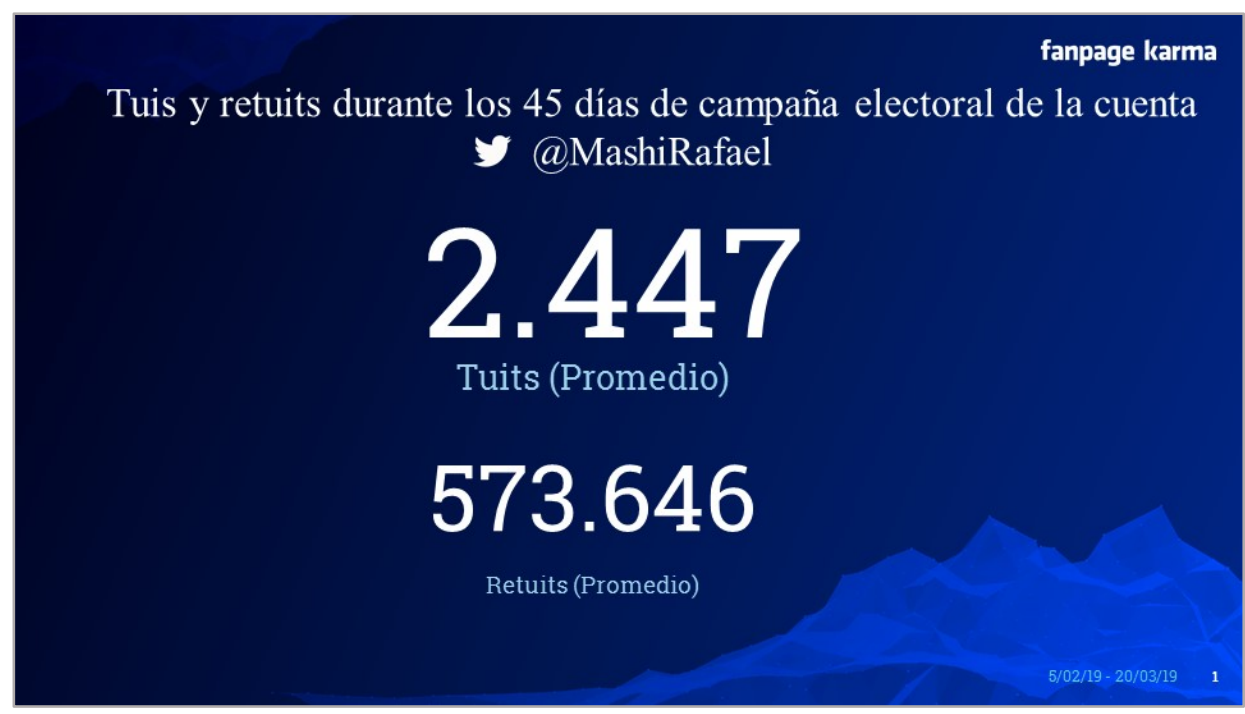

Fuente: Fanpagekarma y rediseñado por los autores

La investigación pretende determinar el grado de influencia que tuvieron las publicaciones en Twitter de un líder externo en la contienda política, enfrentado en un proceso de ruptura partidista que tenía, como objetivo principal, mantenerse en la mente de los electores, dándoles marcos que los referencien a un presente y futuro de recuperación con la lista que respaldaba Rafael Correa o a un futuro de tragedia y destrucción liderado por traidores refiriéndose a Lenin Moreno. Esta relación política de confrontación permite citar a Nicolás Maquiavelo (1513) cuando refiere el posible poder de una oposición al príncipe "si aquellos hombres que, en el principio de un principado eran enemigos del príncipe, no son capaces de mantenerse en su oposición sin necesitar de apoyos, podrá ganarlos el príncipe fácilmente" (p.47). En este caso, el liderazgo de un país está marcado por una oposición digital después de haber dividido el partido Revolución Ciudadana que los catapultó como fuerza política número uno durante diez años en Ecuador. 
La ruptura entre Moreno y Correa es evidente, tanto así que durante los días de campaña la palabra más utilizada en los tuits del expresidente es: "Moreno" (en referencia al actual Presidente del Ecuador Lenin Moreno); que al ser comparados con los hashtags más frecuentes: "\#RevocatoriaMorenoYa" y "\#EICorruptoEsMoreno"; muestran un panorama de oposición al gobierno por parte de Correa en las redes sociales, de este modo los seguidores de su red social @MashiRafael generan interacciones de emociones y sentimientos.

Gráfico 4. Palabras y hashtags en los tuits de Rafael Correa durante la campaña electoral.

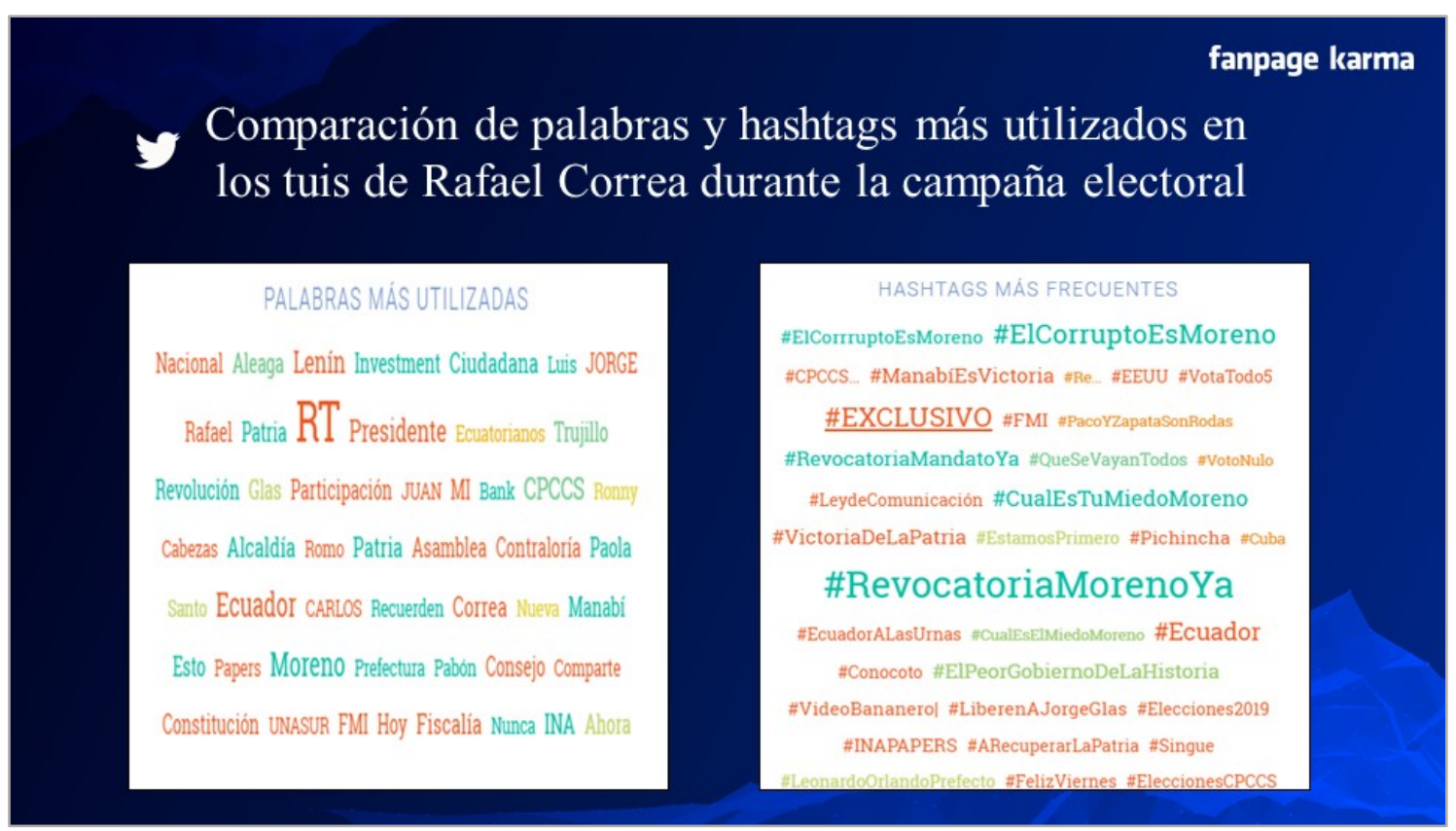

Fuente: Fanpagekarma y rediseñado por los autores

Cuando los sistemas de representación no corresponden a las realidades de los ciudadanos es cuando surge la oposición como un modelo magistrado desarrollado por las culturas griega y romana y que desde su planteamiento según Aristóteles no debe ser ideológico, sino propositivo y es la oposición precisamente quien justifica los lineamientos de democracia en los gobiernos y que debe tener características de "cohesión organizativa, competitividad, ambiente y posibilidad de control de gobierno, especificidad, finalidad ideológica y estrategias partidistas" propuestos por Roberth (Dahl, 1996, p.230) y para consolidar estos criterios referimos los resultados obtenidos 
sobre las 12 prefecturas donde se registraron candidatos, más las alcaldías representativas a las que se inscribieron por el partido objeto de análisis:

- Paola Pavón - Candidata a la Prefectura de Pichincha, ganó con el 22,13\% de votos

- Leonardo Orlando - Candidato a la Prefectura de Manabí, ganó con el 28,51\% de votos

- Pierina Correa - Candidata a la Prefectura del Guayas, obtuvo el segundo lugar con $17,42 \%$ de los votos frente a Carlos Luis Morales que ganó con el $47,87 \%$

- Amanda Ortíz - Candidata a la Prefectura de Santo Domingo, obtuvo el tercer lugar con $11,96 \%$ de los votos, frente a Johana Nuñez que ganó con el 39,02\%

- Héctor Almeida - Candidato a la Prefectura de Imbabura, obtuvo el cuarto lugar con $7,06 \%$ de los votos, frente a Pablo Jurado que ganó con el $43,75 \%$

- Manuel Alvarado - Candidato a la Prefectura de Azuay, obtuvo el cuarto lugar con $12,17 \%$ de los votos, frente a Yaku Pérez que ganó con el 29\%

- Max Iñiguez - Candidato a la Prefectura de Loja, obtuvo el cuarto lugar con 10,31\% de los votos, frente a Rafael Dávila que ganó con el 34,08\%

- Lenin Mera - Candidato a la Prefectura de Santa Elena, obtuvo el cuarto lugar con $8,29 \%$ de los votos, frente a José Villao que ganó con el $27,06 \%$

- Eduardo Zambrano - Candidato a la Prefectura de El Oro, obtuvo el quinto lugar con $8,76 \%$ de los votos, frente a Clemente Bravo que ganó con el 27,73\%

- Humberto Alvarado - Candidato a la Prefectura de Los Ríos, obtuvo el quinto lugar con 11,50\% de los votos, frente a Jonny Terán que ganó con el 26,34\%

- Ramiro Vela - Candidato a la Prefectura de Cotopaxi, obtuvo el sexto lugar con $4,94 \%$ de los votos, frente a Jorge Guamán que ganó con el 31,90\%

- Alexis Sánchez - Candidato a la Prefectura de Tungurahua, obtuvo el octavo lugar con 4,16\% de los votos, frente a Manuel Caizabanda que ganó con el 21,62\% 
En los resultados para alcaldías están referenciados los postulantes de tres ciudades con los datos más relevantes para la investigación.

- Luisa Maldonado - Candidata a la Alcaldía de Quito, obtuvo el segundo lugar con 18,42\% de los votos frente a Jorge Yunda que ganó con el 21,39\%. Caso particular en esta ciudad es el número de concejalías obtenidas para el caso de análisis dado que se obtuvieron 9 de 21 del movimiento Fuerza Compromiso Social (lista $5)$.

- Alexandra Arce - Candidata a la reelección de la Alcaldía de Durán, obtuvo el segundo lugar con $23.93 \%$ del total de votos frente a Dalton Narváez, principal competidor que obtuvo $34,30 \%$.

- Gustavo Jara - candidato a la Alcaldía de Cuenca, obtuvo el quinto lugar con $8,60 \%$ frente a Pedro Palacios ganador con 28,06\% (Consejo Nacional Electoral, 2019)

En Ecuador se eligieron también a los nuevos integrantes del Consejo de Participación Ciudadana y Control Social, entidad autónoma que forma parte y lidera la Función de Transparencia y Control social conformada por siete delegados sociales que no deben poseer ningún tipo de filiación política.

Divididos en tres grupos de representación: 3 varones, 3 mujeres y 1 de representación de minorías, se presentaron a las últimas elecciones 41 candidatos. Rafael Correa de (Soy Revolución Ciudadana, 2019) en un video colgado en redes sociales conminó a elegir los representantes que desde su perspectiva estaban mejor calificados: Walter Gómez. Olindo Nastacuaz, Victoria Desintonio, Graciela Mora, Juan Dávalos y Carlos Espinoza. De estos, solamente Walter Gómez obtuvo el número de votos necesarios para conformar la nueva comisión.

En la actualidad, movimiento Fuerza Compromiso Social mantiene su presencia como partido en el registro electoral del CNE dado que obtuvo 342.473 votos para los candidatos de la lista lo que les representa cumplir el porcentaje mínimo requerido (5\% 
del total del electorado empadronado) para presentarse en futuras contiendas electorales.

\section{DISCUSIÓN}

La visualización de la oposición construida en las redes sociales y los efectos generados en las elecciones seccionales, se esquematizan mediante herramientas de cálculo estadístico digital para medir la influencia de estrategias comunicacionales publicadas en Twitter usados por Movimiento Político "Fuerza Compromiso social" (lista 5), en el caso de Ecuador, la identificación de una oposición de grupos o sectores, no es visible en medios de comunicación ni en espacios sociales, no hay huelgas, no hay denuncias ni quejas, la opinión pública no motiva emociones contrapuestas, no sabemos pero tampoco nos preocupamos por saber, quien ejerce la autoridad en el país informa semanalmente de las acciones y solo unos cuantos a través de redes sociales exigen obras más allá de las palabras.

Karl Popper (1945) hace dos aportes fundamentales para mostrar, justamente, que la democracia significa el ejercicio de la oposición contra cualquier monopolio de la verdad, estos son: i) la raza humana avanza mediante un proceso permanente de solución de problemas a través de 'ensayo y error', sin mayores seguridades, sin certezas, sin profecías y sin la capacidad de formular una especie de 'ingeniería social utópica'; ii) en una democracia, los gobernantes, en especial si son intelectuales, son demasiado propensos a cometer errores y a ostentar ambiciosas pretensiones de poder, y el más sensato y eficaz antídoto para tal enfermedad es el espíritu crítico de la sociedad (Cante Cuervo y Franco Beatriz, 2014, p.9).

Luego de 10 años de un gobierno representado por Rafael Correa, en el que la democracia y oposición estaban en la agenda mediática, aunque satanizada en muchos casos, en los medios convencionales es importante analizar si aún se visualizan esos conceptos en los medios. Para ello, es sustancial mencionar el tema de las temáticas que se abordan en los medios tradicionales, las inferencias que, al respecto, se hacen y cuándo esas interpretaciones distorsionan el sentido de la información. "Los marcos van 
acompañados de inferencias, de modo que cada marco implica algo diferente [...] el marco de simplemente un famoso implica que en estas elecciones no había política partidista y que realmente podría haber ganado cualquier otro famoso" (Lakoff, 2004, p.31).

Las elecciones de marzo 2019 estuvieron marcadas por el involucramiento de una oposición no muy visible pero latente en Twitter donde un expresidente procuraba mantenerse en la palestra pública y generar posturas divididas respecto a la política electoral y los candidatos de la lista que él representaba como la alternativa más favorable.

Pasquino (1988), citado en Guayasamín (2016), al respecto dice desde la Política que: es aquel conjunto de actos y actitudes dirigidos a influir de manera más o menos directa y más o menos legal sobre las decisiones de los poseedores del poder del sistema político o en cada una de las organizaciones políticas, así como en su misma elección, con vistas a conservar o modificar la estructura (y por tanto los valores) del sistema de intereses dominantes. (p.17)

Cuando se analiza la segmentatividad episódica y la saturación en la hipercodificación, se recuerda "una narrativa desbordante y saturada: a nivel de sus lógicas gramaticales en cuanto ensamblaje de fragmentos sobre-codificados, y a nivel de sus tramas y argumentos en cuanto desarrollo narrativo de episodios nucleares limítrofes, tensiones hacia el clímax" como señala (Rivera, 2019, p.287) y, para este caso, las candidaturas que fueron respaldadas por Fuerza Compromiso Social estuvieron ensambladas sobre un discurso de oposición al actual gobierno, destacando siempre narrativas emocionales que apelaban a un pasado mejor.

Por otro lado, cuando se habla de ciberdemocracia concebimos al recurso del Internet como la máxima para difundir los discursos o las propuestas, pues lo vemos como un espacio en el que todos pueden participar y darse a conocer, se configura como un espacio democrático en el que se visualiza la participación y el ejercicio de la política. La democracia electrónica donde: 
Se hace hincapié en dos aspectos: en primer lugar, la disposición de los individuos a adquirir informaciones tanto como las posibilidades de que las mismas impliquen una profundización de la participación y una mejora de la democracia; en segundo lugar, el riesgo - por ciertas modalidades propias de este tipo de comunicación- de reforzar la tendencia de concebir la política como gestión, como elección de quien cumple con más eficacia los servicios que se demandan (Martino, 2003, p.389).

La espectacularización de la información que se consume en redes ha sido también un factor que desencadenó las interacciones en Twitter y otras redes en las que todos opinan, informan y crean, como señala (Baiget, 2018) sobre los ejes de la espectacularización en redes sociales. En la misma línea, este autor refiere la aplicación de los medios en la consolidación de estas como un "sistema comunicativo híbrido" (Chadwick, 2013) lo que ha supuesto un impulso hacia una mayor espectacularización de la política. La "espectacularización de la política" en redes sociales ha sido tomada por los actores de la comunicación política (ciudadanos, partidos políticos y medios de comunicación) como un espacio para posicionar y legitimar su discurso. Al interpretar lo referido por Mcluhan y Nevitt, en 1972: “la tecnología electrónica permite al individuo asumir a la par, roles de productor y de consumidor de contenidos" Ahora los individuos se sienten libres de publicar, crear y compartir su propio discurso (García-Guerrero, 2019, p.232).

No obstante, eso no se aprecia en los medios ecuatorianos. La espectacularización en el período de campaña se traslada a las redes sociales. Twitter como recurso de difusión de los mensajes incitadores o buscadores de respuesta. Como señala Rivera (2019), en la televisión se expone la importancia de las representaciones sociales y la aceptación o negación que los públicos puedan tener de un mensaje, o en este caso de un candidato.

En las modernas democracias no son los individuos sino, más bien, los grupos los protagonistas principales de la política. En tal régimen imperan grupos de presión como gremios y grupos económicos, empresas, partidos políticos, sindicatos; y a veces movilizaciones $o$ acciones colectivas populares de sectores 
tradicionalmente excluidos y marginados de las decisiones políticas (Cante Cuervo y Franco Beatriz, 2014, p.11).

Correa toma a su red social Twitter como el espacio libre de intermediadores para poder ejercer su poder comunicativo sin ninguna barrera:

"En Twitter, Correa tiene la posibilidad de medir las reacciones de otros usuarios y las tendencias de lo que se está hablando a nivel mundial y nacional. Con base en esa información, escribe sus propios tweets. En otras ocasiones, ha sido él quien genera las tendencias en la red social. Adicionalmente, mediante Twitter, Rafael Correa trata de mantener viva la Revolución Ciudadana y que la gente continúe reconociéndolo como el líder que gestó esa lucha y generó grandes cambios para el país" (Gordillo Tejada, 2019, p.100).

Rafael Correa usa políticamente su red social Twitter, permitiéndole en muchas ocasiones ser quien ponga tema en la agenda mediática y política del Ecuador. Además, refuerza la creación de su "Mito de la Revolución Ciudadana" (Ávila Nieto, 2012, p.140) generado durante su gobierno como un líder e imagen de un cambio que aún está presente en el imaginario de muchos ciudadanos.

Para Lenin Moreno, marcar una separación del correismo fue generar una ruptura en su discurso tomando en cuenta que llegó al poder cobijado por el exmandatario y sus simpatizantes. Albán Gallo y Velazco (2018), afirman que "en Moreno las categorías patria, ciudadanía, democracia, partidocracia y revolución pierden sentido en su totalidad" (p.12). Pero esta ruptura fue más allá de los discursos y Moreno cambió ideológicamente las líneas de su gobierno, siendo catalogado por Correa como un "traidor" quien hace uso de las redes sociales para emitir su criterio y de alguna forma construir la opinión pública, conforme señala Pillajo Paredes (2017), "las redes sociales se han convertido en la nueva ágora de la sociedad o en nuevo escenario deliberación pública donde es posible debatir y expresarse libremente, amplificando la voz a los políticos y ciudadanos, convirtiéndose en un terreno de disputa" (p.120). Esta lucha de poder entre Moreno y Correa se evidenció en redes sociales, especialmente en Twitter. 
Moreno también hizo uso de las redes sociales y en sus discursos emitió mensajes como "la gente abandonará su comportamiento ovejuno y va a empezar a respirar verdaderamente libertad" (Labarthe y Saint-Upery, 2017, p.31). Esto fortaleció la ruptura con los militantes y empezó a marcar pistas que señala Minervini (2007), “[...] el discurso de Moreno está repleto de segmentos yuxtapuestos y coordinados, cuya función no persigue el esclarecimiento de una idea o de una información, sino enfatizar en esta" (p.66). En las redes sociales las tendencias en Twitter se vuelven un termómetro de la sociedad (Pillajo Paredes, 2017, p.134). Twitter está catalogada como una red social informativa con cerca de 500 millones de usuarios alrededor del mundo y las disputas llevadas en el terreno digital se hacían eco en la agenda pública y mediática.

"Internet es un instrumento para promover la democracia. Por su fácil acceso a información política, permite a los ciudadanos ser casi tan bien informados cuantos sus líderes" (Castells, 2002, p.138). La realidad difiere bastante de esto, en Ecuador en las redes sociales se encuentran los "ataques" entre los partidos políticos, buscando desacreditarlos en vez de preocuparse por tener justamente la interactividad con los usuarios de estas redes y obtener "puntos a favor" a través de las mismas (López y Cabrera Silva, 2014, p.67).

Dentro de este contexto de "ataques", se desarrolló la campaña política de las elecciones seccionales para autoridades locales (prefectos, alcaldes, concejales, presidentes y vocales de juntas parroquiales), además de la designación de los Consejeros del Consejo de Participación y Control Social en Ecuador. La desacreditación dentro de las redes sociales fue un factor que no faltó dentro de la campaña electoral. Para Correa el espacio mantenido en redes sociales durante estos años representaba una mayor influencia sobre sus seguidores, así lo explica el estudio de Báez Urrutia (2018) "La estructura semántica del discurso de Rafael Correa puede influenciar en la manera de pensar de muchos de sus seguidores convenciéndolos así de quienes son los verdaderos corruptos y quienes tienen las manos limpias" (p.64) desde una perspectiva emocional, Correa se muestra como el político digital que no ha cambiado su ideología. 
La campaña en redes sociales descubre a "nuevos políticos" según López y Cabrera Silva (2014) que: "no debe solo parecer, sino ser ya que las redes serán la carta de presentación de sinceridad y autenticidad" (p.68). Entonces, el desarrollo de una campaña política dentro de las redes sociales deberá llegar a la emotividad y conocimiento del usuario de redes. Sin embargo, en la democracia moderna ecuatoriana, no fueron los grupos los que ejercieron presión sino una persona que, a través de la red social Twitter, motivó la elección hacia sus representados, buscando siempre influir en las decisiones políticas de los electores frecuentemente consumidores de nuevas formas de comunicación como Twitter.

Las dinámicas de la actividad política en la que estar presente en los medios es fundamental para la campaña se mantiene, puesto que siguen siendo los atriles donde se dan a conocer los planes de trabajo de los candidatos y Sánchez, García, y Silvestre (2010) ya lo destacaban al escribir "La imagen del candidato y su venta constituyen un objetivo central de las campañas, y para ello se recurre a un complejo aparato propagandístico controlado por los especialistas en marketing electoral” (p.55).

\section{CONCLUSIONES}

Sobre los candidatos, "Fuerza Compromiso Social" movimiento político (Lista 5) fundado por un exministro del gobierno de Rafael Correa sirvió de colchón para la inscripción de los candidatos correístas; en la investigación se priorizaron los resultados de las candidaturas de Prefecturas y miembros del Consejo de Participación Ciudadana y Control Social de por ser las más representativas mediáticamente.

Las nuevas tecnologías de la información y comunicación constituyen alternativas de comunicar para activistas, políticos y ciudadanos. Los medios digitales en la investigación tienen un perfilamiento afín al partido que respalda Rafael Correa y de ahí los discursos relacionados o de línea preferencial, sin duda, ante la postura mediática tradicional donde no se prioriza el tema establecido en el estudio. Es visible la postura de la oposición, pero tampoco existe mayor información que permita argumentar la postura del gobierno. La formación de los discursos en redes sociales, permite al ciudadano sentir la libertad de creer y compartir lo que le gusta, bajo una realidad 
subjetiva para Martín Rojo (1996) “los discursos no reflejan la realidad, no son un espejo fiel de ésta" (p.1), la autora también acota que sin importar que el discurso sea real o no, el individuo buscará ser parte de estos espacios para no sentirse excluido.

"El poder radica en la soberanía popular" recalcaba Thomas Jefferson (Bilbao, 2013), siendo la participación de los ciudadanos el eje central para elegir la representación política, que tendrá como misión dirigir las estructuras institucionales creadas para la organización de los territorios, en la investigación es notoria la participación de los ciudadanos y sobre todo los espacios que se buscan o los recursos que se generan para participar sobre la cosa política y para Rafael Correa el uso de redes sociales permitió desafiar al poder mediático de los medios tradicionales y con un discurso emotivo o de confrontación mantiene vivo el interés de los ciudadanos en lo que dice o la postura que toma de acuerdo a un tema. Según Villavicencio Salvador (2017): “[...] fue capaz de conectarse con la gente desafiando a poderes tradicionales del Estado [...]" (p.112).

Los medios de visualización de las candidaturas no son equitativos y se construyen a través de las redes sociales. En el caso del movimiento político FCS, éste se fortalece en los espacios digitales. Es necesario ampliar el análisis sobre estos recursos para determinar su efectividad y determinar si efectivamente se constituyen como espacios de oposición. Interpretando el análisis que hace Greppi (2014) sobre Pasquino es posible inferir que:

"no tendremos una democracia a la altura y que no la tendremos nunca si no cambian los ciudadanos, se necesitan ciudadanos que posean una información política adecuada, que alimenten la socialización de la democracia. La participación debe consistir en la posibilidad de incidir, esto es, tomar parte en las decisiones y cambiarlas cuando proceda" (p.5).

Tomando en cuenta que Internet y, más aún, las redes sociales permitieron que el ciudadano cree su propio discurso, fueron los políticos quienes cambiaron el tradicional "balcón o plaza" por una red social. En Ecuador, Rafael Correa es uno de los políticos que más uso da a la red social Twitter con cerca de 3.4 millones de seguidores. Referencia Gordillo Tejada (2019) "el exmandatario ecuatoriano conoce el poder y la 
fuerza que tiene su discurso en Twitter, al punto de que sus mensajes son replicados en los medios tradicionales de comunicación y generan noticia, no solo en el ámbito nacional, sino también internacional (...)” (p.99).

Pierina Correa, candidata que no fue favorecida en la contienda electoral de Prefectos por la provincia del Guayas, sobre los resultados de las elecciones seccionales 2019, refirió a los medios:

"señaló que tuvieron obstáculos para participar en las elecciones tras indicar que a 60 días de las mismas no tenían movimiento y que en plenos comicios un 30\% de la población creía que aún eran representados por la listas 35 (Alianza PAÍS) y otro $30 \%$ no sabía que existían, que estuvieran participando o por qué lista lo estaban haciendo" (Diario El Universo, 2019).

En la actualidad, movimiento Fuerza Compromiso Social mantiene su presencia como partido en el registro electoral del CNE dado que obtuvo el porcentaje mínimo requerido $5 \%$ de votos del total del electorado empadronado, y con ese porcentaje, el partido está habilitado para presentarse en futuras contiendas electorales. Resta investigar si en las elecciones del 2021 los correístas postularán a los comicios de elección nacional.

\section{REFERENCIAS BIBLIOGRÁFICAS}

Báez Urrutia, M. (2018). Rafael Correa en Twitter: análisis crítico del discurso de la cuenta@MashiRafael. Quito.

Albán Gallo, E., y Velasco, M. (2018). El discurso político de posesión en Ecuador, de la revolución ciudadana de. Tsafiqui, 1-13.

Ávila Nieto, C. (2012). El mito como elemento estratégico de comunicación política: aplicación del modelo de Barthes al caso ecuatoriano. Cuadernos.Info, 31, 139-150. doi: $10.7764 /$ cdi.31.447

Baiget, T. (2018). El profesional de la información. 
Bilbao, J. (2013). La democracia según Thomás Jefferson. Jot Down El Sur de Picaso, 17.

Cante Cuervo, F., y Franco Beatriz. (2014). Renovadas formas de hacer oposición. Rosario, Argentina: Editorial Universidad del Rosario. Recuperado de http://ebookcentral.proquest.com/lib/utnortesp/detail.action?doclD=4760714

Consejo Nacional Electoral. (2019). cne.gob.ec. Obtenido de Sitios web para consulta de candidaturas y planes de gobierno para elecciones 2019:

https://candidatos2019.cne.gob.ec/

Dahl, R. (1996). Political Opposition in Western Democracies. New Haven Yale: University Press.

Dahl, R. (1997). La Poliarquía participación y oposición. (I. 8430917985, Ed.) Barcelona: Tecnos.

Diario El Universo. (2019). Diario El Universo. Recuperado de https://www.eluniverso.com/noticias/2019/04/02/nota/7265561/correistas-esperancompetir-elecciones-2021-movimiento-prestado

García , G. (2017). Estrategia y publicidad electoral: los spots de campaña en Andalucía 2008 y 2012. REDALyC, 161-186.

García Jurado, R. (2000). Fundamentos de la Legitimidad. Estudios Políticos, 129-153. García, M. (2016). Actitudes hacia la participación política en habitantes de la ciudad de Quito. Repositorio Universidad Salesiana, 28-36.

García-Guerrero, J. (2019). Redes Sociales e interés político: frecuencia con la que se comparte información sin confirmar en Quito. Ícono, 2(14), 231-253. doi:10.7195/ri14.v17i2.1308

Gordillo Tejada, M. F. (2019). Usos y prácticas del poder político de twitter: análisis del discurso del expresidente de la república del Ecuador, Rafael Correa, a través de la 
cuenta@MashiRafael y su influencia en las percepciones de la imagen gubernamental. Quito.

Greppi, A. (2014). StuDocu, Universidad Carlos III de Madrid. Studocu.com. Recuperado de https://www.studocu.com/es/document/universidad-carlos-iii-de-madrid/filosofiapolitica/resumenes/resumen-democracia-elecciones-partidospasquino/333691/view?shared $=\mathrm{u}$

Guayasamín, D. (2016). Actitudes hacia la participación política en habitantes de la ciudad de Quito, en el periodo agosto 2015 a marzo 2016. Quito: Repositorio Institucional de la UPS. Recuperado de http://dspace.ups.edu.ec/handle/123456789/13443

Labarthe, S., y Saint-Upery, M. (2017). Leninismo versus Correísmo: la tercera vuelta en Ecuador. Nueva Sociedad, 272, 29-42.

Lakoff, G. (2004). No pienses en un elefante, lenguaje y debate político. Madrid: Chelsea Green Publishing.

López, M., y Cabrera Silva, T. (2014). Campaña Política a través de redes sociales. ComHumanitas, 65-72. Recuperado de https://dialnet.unirioja.es/servlet/articulo?codigo $=5845775$ Maquiavelo, N. (1513). El Príncipe. Alicante, España: Biblioteca Virtual Miguel de Cervantes.

Martín Rojo, L. (1996). El orden social de los discursos. Universisda Autónoma de México. Recuperado de http://ru.iis.sociales.unam.mx:8080/jspui/handle/IIS/5548 Martino, B. (2003). Ciberdemocracia ¿Ciberoptimismo? Análisis de algunos límites de los usos de Internet para el gobierno electrónico. Millcayac. Anuario de Ciencias Políticas y Sociales, 1(1), 381-398. Recuperado de https://bdigital.uncu.edu.ar/app/navegador/?idobjeto=131 
Parredes Goicochea, D. F. (2015). La acción política en Karl Marx. Una lectura a partir deHannah Arendt y Maurice Merleau-Ponty. (Tesis doctoral ). Bogotá: ED382 Universidad de Paris Diderot. Recuperado de http://www.bdigital.unal.edu.co/49430/ Pillajo Paredes, A. (2017). Interacción en twitter: análisis de la cuenta de twitter del Presidente de la República Vicente Rafael Correa Delgado @MashiRafael. Universidad de las Américas. Quito: Universidad de las Américas. Recuperado de http://dspace.udla.edu.ec/handle/33000/7558

Rivera, F. (2019). De jack el destripador al síndrome de scarface -representaciones sociales, "real t.v» y tele-fisiognómica.

Sánchez, G., García, H., y Silvestre, D. (2010). Comunicación y democracia : Reflexiones sobre el nuevo escenario mediático y legal de los medios de comunicación en méxico: reforma electoral, televisa y la ley de transparencia. México: UNAM.

Sartori, G. (2014). Ensayo ¿'Tiene futuro la democracia? Clarín.com. Recuperado de http://www.anepág.edu.uy/historia/clases/clase10/cuadros/11_art_sartori.pdf

Soy Revolución Ciudadana. (2019). Soy Revolución Ciudadana, Candidatos al Consejo de Participación Ciudadana. Recuperado de https://www.youtube.com/watch?v=NTS8bQ2L90s

Villavicencio Salvador, Y. (2017). ANÁLISIS DEL CONTENIDO COMUNICACIONAL EMITIDO EN LA CUENTA @MashiRafael EN LA RED SOCIAL TWITTER EN EL PERIODO DE ELECCIONES PRESIDENCIALES DEL ECUADOR AÑO 2017. Guayaquil. Recuperado de http://repositorio.ug.edu.ec/bitstream/redug/36899/1/EMPASTADO FINAL YUJAN VILLAVICENCIO.pdf 